\title{
Various Vaccination for Coronavirus
}

\section{Mitra $\mathbf{M}^{*}$}

Department of Alumnus with Electrical Engineering, University of Bridgeport, Bridgeport, United States

*Corresponding author: Manu Mitra, Department of Alumnus with Electrical Engineering, University of Bridgeport, Bridgeport, United States, Email: mmitra@my.bridgeport.edu

\section{Editorial}

Volume 5 Issue 1

Received Date: April 17, 2020

Published Date: June 12, 2020

DOI: $10.23880 /$ nnoa-16000184

\section{Introduction}

Coronavris comes from its structure which appears more like a crown when viewed through electron microscope. It is transmitted through air and other medium; it can infect both lower airways and upper airways of respiratory system causing severe respiratory illness which is called Severe Acute Respiratory Syndrome (SARS) and other complications in humans [1]. COVID-19 has been identified as a new strain of Beta coronavirus with approximately $70 \%$ genetic similarity to SARS-CoV [2].

There are many attempts made for coronavirus vaccines and drugs for antiviral treatment.

Index Terms - Coronavirus, RNA, Ribonucleic acid, Protein, vaccination, immunization, Middle East Respiratory Syndrome, Severe Acute Respiratory Syndrome, MERS, SARS

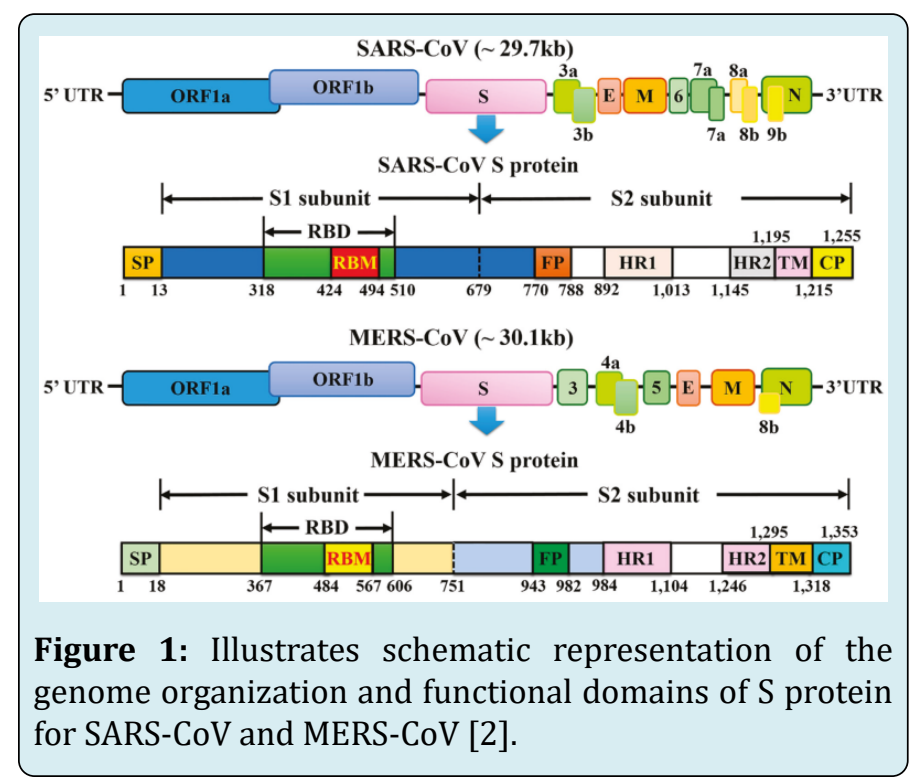

\section{Coronavirus Vaccination for Covid-19}

School of Medicine at University of Pittsburgh declared a possible vaccination against SARS COIVID-19 pandemic. When it was tested in mice; vaccine was delivered through fingertip sized patch, it produces antibodies specifically to SARS COVID-19 at quantities that supposed to be sufficient for neutralizing the virus.

Related to the experimental mRNA vaccine which scientist call as "PittCoVacc" in short termed as Pittsburgh Coronavirus Vaccine. It is lab made pieces of viral protein to increase immunity. It's the similar way the flu shots work. Scientist also used novel method to deliver the drug called microneedle array, to increase potency. This array is the size of fingertip patch of 400 tiny needles that carries the spike protein pieces into the skin. Where the immune reaction is strongest [3,4].

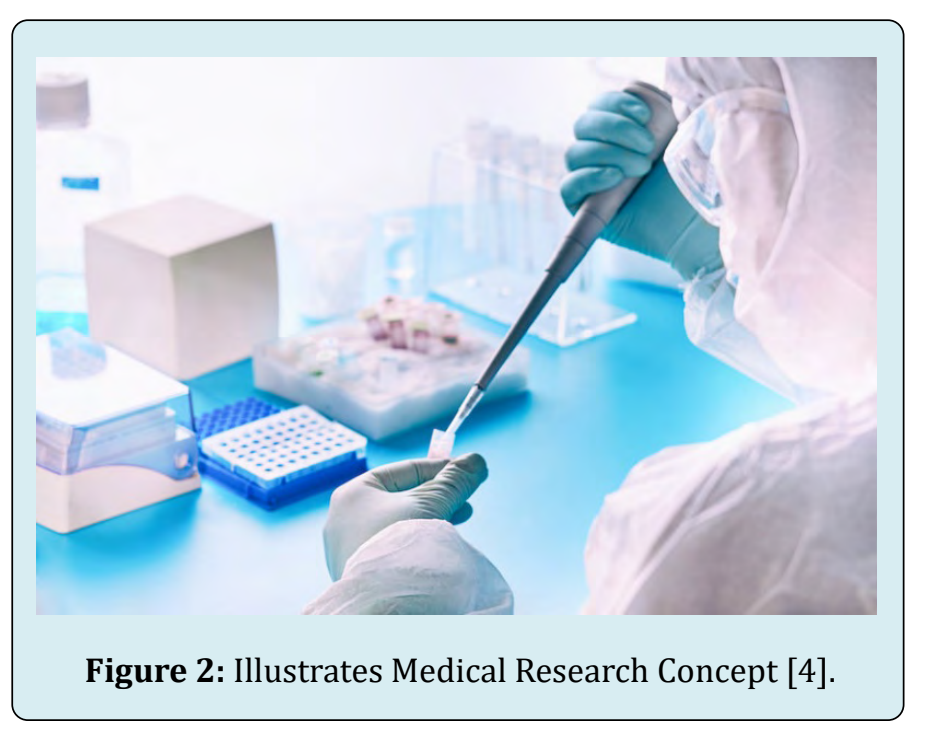




\section{Safe and Efficient Sars Coronavirus Vaccine}

In this study, scientists conducted an experiment on live attenuated (weakened) viral vaccines that are considered safe as long as their "reversal" to a virulent (disease causing) virus is prevented.

Scientists addressed the question of steadiness of the vaccine contender. To perform this, they propagated SARS Cov $\Delta \mathrm{E}$ virus for a number of generations in cell lines in mice and they discovered that over time - the virus accumulates mutations and reverts to a virulent phenotype. After studying collection of mutants, they were able to disclose the molecular basis of the reversion; The E-protein that contains a motif called a PDZ binding - It is a protein recognition sequence that modulates cellular pathways that is important for viral replication, dissemination in the host and pathogenesis; obliviously to compensate for removal of motif with deletion of E protein. To avoid such compensation and reversal to virulence and instead of deleting entire gene, they introduced minor deletions in the $\mathrm{E}$ gene that did not terminate its PBM.

Scientists concluded that comprehending the molecular mechanisms that leads to pathogenicity and in vivo evaluation of vaccine genetic stability is promising for rational design of SARS CoV vaccine $[5,6]$.

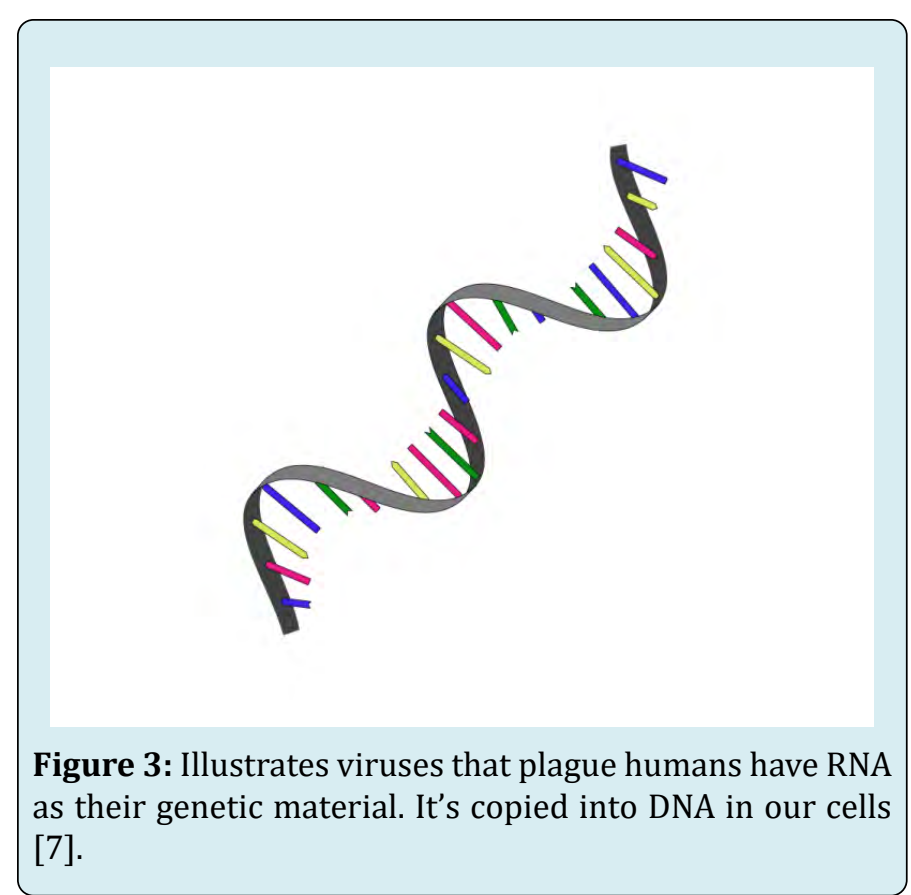

\section{Testing Sars Cov-2 Vaccine}

Scientists and researchers from South Australian working with Oracle Cloud technology and vaccine technology discovered a novel vaccination for COVID-19 pandemic.

Modern cloud based technology provided by Oracle allowed the team members to vividly speed up the ability to analyze the COVID-19 virus and use this information to design vaccine contender. As soon as genomic sequence of COVID-19 became available in January, they straightaway used it to develop SARS coronavirus vaccine to characterize the vital viral attachment molecule called spike protein. They used computer simulations of the spike protein and its human receptor ACE2 to recognize how the virus was infecting human cells and then they were able to design vaccine to block this replication process [8].

\section{Breakthrough in Coronavirus Vaccine Design}

Scientists from University of Texas at Austin made a significant breakthrough toward developing a vaccine for the 2019 novel coronavirus by making the first 3D atomic scale map of the part of the virus that attaches and infects human cells.

Mapping the spike protein is a vital and critical step so that researchers around the world can develop vaccines and antiviral drugs to combat the virus. Jason and his team members designed a new technique to combat coronavirus including SARS CoV and MERS CoV. They developed various techniques for locking coronavirus spike proteins into a form that made easier to analyze, prioritize and could effectively turn it to contender for vaccines. This study and analysis gave them an advantage over other research teams understanding novel virus. The molecule that the team created and for which they obtained a structure that represents only extracellular part of the spike protein, but is enough to produce an immune response in people and hence serve as a vaccine $[9,10]$.

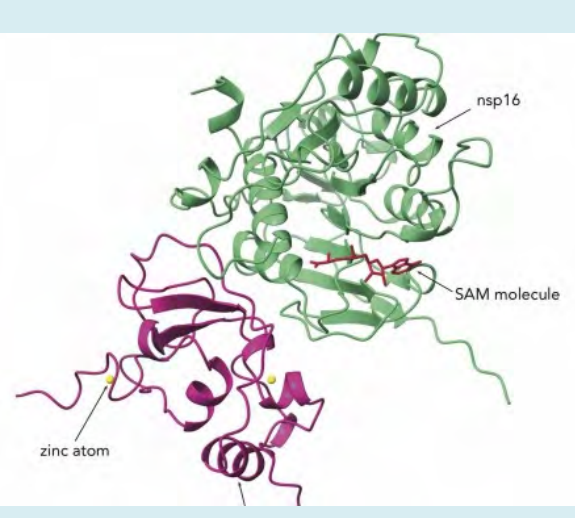

Figure 4: Illustrates A new potential drug target has been identified in SARS CoV-2 - the virus that causes COVID-19 - by scientists who say multiple drugs will likely be needed to respond to the pandemic [11]. 


\section{Covid-19 Development of Vaccine}

Researchers at the Hong Kong University of Science and Technology (HKUST) has recently made significant discovery in classifying a set of potential vaccine targets for SARS CoV (coronavirus) providing development leads for guiding experimental efforts towards vaccine development.

In spite of similarities between SARS CoV and SARS $\mathrm{CoV}-2$, there is genetic variation between the two and it is non-obvious. If epitopes that produce an immune response against SARS CoV will more likely be effective against SARS CoV-2. They found that roughly $20 \%$ of SARS CoV epitopes map identically to SARS CoV-2 and are promising contender for vaccination. Their objective was to assist initial phase of vaccine development by providing specific epitopes that may potentially considered for vaccination for COIVID-19. In general to work in global effort seeking vaccination available for COVID-19 made available and rapidly shared by scientific community to comprehend this new virus and come up with effective interventions $[12,13]$.

\section{Conflicts of Interest}

There are no conflicts of interest as per Author's point of view.

\section{References}

1. Guo H, Guangxiang, Gao S (2020) The new coronavirus outbreak in China may have originated in snakes. PhillyVoice.

2. Coronavirus (2003) In Wikipedia, the free encyclopedia.

3. Eun Kima, Geza Erdosb, Shaohua Huanga, Thomas W Kennistona, Stephen C Balmertb, et al. (2020) Microneedle array delivered recombinant coronavirus vaccines:Immunogenicity and rapid translational development. EBioMedicine 55: 102743.
4. University of Pittsburgh (2020) COVID-19 vaccine candidate shows promise, research shows. ScienceDaily.

5. Jose M Jimenez-Guardeño, Jose A Regla-Nava, Jose Nieto-Torres, Marta L DeDiego, Carlos CastañoRodriguez, et al. (2015) Identification of the Mechanisms Causing Reversion to Virulence in an Attenuated SARS-CoV for the Design of a Genetically Stable Vaccine. PLOS Pathogens 11(10): e1005215.

6. PLOS (2015) Towards a safe and efficient SARScoronavirus vaccine: Mechanism and prevention of genetic instability of a live attenuated virus.

7. Lewis $\mathrm{R}$ (2020) COVID-19 vaccine will close in on the spikes. PLOS Blogs Network | Diverse perspectives on science and medicine.

8. Flinders University (2020) Vaccine candidate against SARS-CoV-2 being tested.

9. Daniel Wrapp, Nianshuang Wang, Kizzmekia S Corbett, Jory A Goldsmith, Ching-Lin Hsieh, et al. (2019) Cryo-EM structure of the 2019-nCoV spike in the prefusion conformation.

10. University of Texas at Austin (2020) Breakthrough in coronavirus research results in new map to support vaccine design.

11. Paul M (2020) new drug target found for COVID-19. Northwestern Now News.

12. Syed Faraz Ahmed, Ahmed A Quadeer, Matthew R McKay (2020) Preliminary Identification of Potential Vaccine Targets for the COVID-19 Coronavirus (SARS-CoV-2) Based on SARS-CoV Immunological Studies. Viruses 12(3): 254.

13. Hong Kong University of Science and Technology (2020) COVID-19 vaccine development. 